Results Girls, but not boys, hospitalised for infections at least twice in the first 6 months of life had pubertal onset at about 8 months older (mean 10.3 years), time ratio 1.08 (95\% CI: 1.04 to 1.12), than those without such hospitalisations (9.6 years) adjusted for infant characteristics and socio-economic position (sex interaction $\mathrm{p}$ value 0.02 ). There were no such associations for infections at 6 months to $<8$ years. Growth did not mediate the association.

Conclusion Early infectious morbidity in girls may be associated with later puberty, perhaps via suppression of the gonadotropic axis. Fewer infections in early life with economic development may be an additional factor contributing to earlier puberty.

\section{P2-149 BEHAVIOURAL RISK FACTORS FOR CVD AMONG ADULT SLUM DWELLERS IN NAIROBI}

doi:10.1136/jech.2011.142976i.84

C Kyobutungi, ${ }^{*}$ S Oti. African Population and Health Research Center, Nairobi, Kenya

Introduction About $80 \%$ of deaths from non-communicable diseases (NCD) occur in developing countries and behavioural risk factors that underlie the burden of major NCD are on the rise in SSA. However, most research and development work has focused on communicable diseases such as malaria and HIV/AIDS while neglecting NCD. This study aims to contribute to the state of knowledge on the prevalence of modifiable risk factors for NCD specifically cardiovascular diseases in the adult population of two slums in Nairobi, Kenya.

Methods A cross-sectional survey among 5190 adults selected by stratified random sampling in two Nairobi slums covered. Data were collected on behavioural risk factors for cardiovascular diseases including self-reported tobacco and alcohol use, dietary habits and physical activity.

Results About $19 \%$ of respondents had ever used tobacco, and $12 \%$ were current users. Males were 20 times more likely to be current tobacco users than females. Majority of respondents (85\%) reported sufficient physical activity though large sex differences were observed. Females were three times more likely to be inactive than males. Less than half of respondents consumed sufficient fruits and vegetables while a little over a third had high salt consumption. Ten percent of respondents were current alcohol users of which, more than a third were frequent heavy drinkers.

Conclusion We found high levels of behavioural risk factors for NCD, specifically high alcohol and tobacco use and poor dietary habits albeit with marked gender differentials. Targeted intervention programmes that promote healthy lifestyles among the urban poor are recommended.

\section{P2-150 RESULTS OF THE 22-YEARS STUDY OF LEUKAEMIA IN CHERNOBYL ACCIDENT CLEAN-UP WORKERS IN UKRAINE}

doi:10.1136/jech.2011.142976i.85

A Romanenko, B Ledoshchuk. . Research Center for Radiation Medicine, Kyiv, Ukraine

Introduction The study was conducted to determine whether the leukaemia incidence rate in Chernobyl Nuclear Power Plant catastrophe clean-up workers depended on the year of the emergency work and the period after irradiation.

Methods All cases collected were verified. The analysis was performed for workers in 1986 and 1987 by periods of observation of 1987-1991 and 1992-1996.

Results Leukaemia RR in workers of 1986 vs workers of 1987 in 1987-1991 was 2.32 (95\% CI 1.19 to 4.70). No significant differences were identified in 1992-1996. The results of these studies were the beginning of the project "Leukaemia" in collaboration with the Cancer Institute of the U.S. The project used modern methods for epidemiological studies and created a database of case and controls. Further analysis is based on 71 cases of histologically confirmed leukaemia diagnosed in 1986-2000 and 501 residencematched controls selected from the same cohort Study subjects or their proxies were interviewed about their clean-up activities and other relevant factors. The excess RR (ERR) of total leukaemia was 3.44 per Gy $(95 \%$ CI 0.47 to $9.78, \mathrm{p}<0.01]$. We found a similar doseresponse relationship for chronic and non-chronic lymphocytic leukaemia $[E R R=4.09$ per Gy $(95 \% \mathrm{CI}<0$ to 14.41$)$ and 2.73 per Gy (95\% CI $<0$ to 13.50 ), respectively]. We found a linear dose-response relationship between Chernobyl-related radiation exposure among cleanup workers and their risk of leukaemia.

Conclusion The project plans to extend the case-control study to ascertain cases for another 6 years (2001-2006).

\section{P2-151 DETERMINANTS OF INCIDENT AND PERSISTENT FINGER JOINT PAIN DURING A 5-YEAR FOLLOW-UP AMONG FEMALE DENTISTS AND TEACHERS}

doi:10.1136/jech.2011.142976i.86

H Ding, S Solovieva, P Leino-Arjas. ${ }^{*}$ Finnish Institute of Occupational Health, Helsinki, Finland

Introduction To investigate the association of radiographic finger osteoarthritis $(\mathrm{OA})$, hand use, and lifestyle factors with incident and persistent finger joint pain among female dentists and teachers.

Methods Random samples of female dentists $(n=295)$ and teachers $(n=248)$ aged $45-63$ years were examined for the presence of finger joint $\mathrm{OA}$ by radiography. Body weight was measured. Information on finger joint pain during the past 30 days, height, smoking, and leisure-time hand activity was collected by questionnaire. Five years later, 482 women (ie, $89 \%$; $65 \%$ still occupationally active) responded to a survey on finger joint pain.

Results The incidence and persistence of finger joint pain were higher among the subjects with OA compared to those without. The RR of incident pain in the $1-3^{\text {rd }}$ fingers was 1.8 (95\% CI 1.2 to 2.7) in the right hand and 3.0 (2.0 to 4.6) in the left, allowing for age, occupation, and lifestyle factors. The corresponding figures for the $4-5^{\text {th }}$ fingers were 2.3 (1.4 to 3.8) in the right and 1.9 (1.1 to 3.5 ) in the left hand. Regarding persistent pain, the RRs varied between 2.4 and 5.4. Body mass index, smoking, or leisure-time hand activity were not associated with pain. The dentists tended to have a higher incidence of pain in the $1-3^{\text {rd }}$ fingers of the right hand than the teachers.

Conclusion Radiographic finger joint $\mathrm{OA}$ was a significant determinant of both persistent and incident finger joint pain in a 5-year follow-up among middle-aged women. Hand use may modify the association between radiographic $\mathrm{OA}$ and finger joint pain.

\section{P2-152 ASSESSING MENTAL HEALTH AMONG WOMEN: THE HEALTHY COMMUNITY COUNCIL 2011 ASSESSMENT}

doi:10.1136/jech.2011.142976i.87

${ }^{1} \mathrm{~K}$ Lewis, ${ }^{*} \mathrm{C}$ Reeves. ${ }^{1}$ James Madison University, Harrisonburg, Virginia, USA ${ }^{2}$ Rockingham Memorial Hospital, Harrisonburg, Virginia, USA

Introduction The Healthy Community Council (HCC) is a nonprofit community-based network composed of over 100 local stakeholders representing both public and private organisations within the community. The HCC has been working to improve the health of the community by conducting needs assessments, identifying priorities and assisting in the implementation of programs and 